\title{
Using Microfluidics to Observe the Effect of Mixing on Nucleation of Protein Crystals
}

\author{
Delai L. Chen, Cory J. Gerdts, and Rustem F. Ismagilov* \\ Department of Chemistry, The University of Chicago, 5735 South Ellis Avenue, Chicago, Illinois 60637
}

Received April 8, 2005; E-mail: r-ismagilov@uchicago.edu

This paper analyzes the effect of mixing on nucleation of protein crystals. Nucleation is an important aspect of protein crystallization. ${ }^{1}$ In-depth research has been conducted leading to insights into the mechanism of crystal nucleation as well as novel methods to control $\mathrm{it}^{1,2}$ (e.g., work on nucleation at the liquid-liquid-phase boundary and on levitated droplets), but the effect of several factors affecting nucleation is not well understood. Mixing is proposed to be responsible for uncertainties in batch protein crystallization. ${ }^{3}$ Mixing is also important in the nucleation of small molecule crystals ${ }^{4}$ and selectivity of organic reactions, ${ }^{5}$ but its effect on nucleation of protein crystals has not been studied experimentally. One barrier to this study is the difficulty of controlling and monitoring mixing, especially when using manual or robotic pipets. Crystal nucleation is a stochastic process, and to obtain statistically significant data, studies of nucleation necessitate a large number of experiments. The amount of the protein and labor required presents another barrier.

Here we show that a plug-based microfluidic system ${ }^{6}$ is suitable for observing mixing effects in crystal nucleation. The system is capable of setting up hundreds of crystallization experiments in a short period of time, ${ }^{7}$ requiring little labor and $\sim 1 \mu \mathrm{L}$ samples of protein solutions. It relies on $\mathrm{nL}$-volume aqueous plugs in a waterimmiscible, fluorinated carrier fluid, where each plug acts as a microreactor in which a crystallization trial takes place. Mixing by chaotic advection in the system is well-controlled and characterized. ${ }^{8}$ Chaotic advection has been pioneered in single-phase microfluidic devices. ${ }^{9}$ The use of two-phase flows in plugs is attractive because it can transport solids, ${ }^{10}$ such as precipitates that might arise during crystallization experiments.

Mixing experiments were carried out by combining protein (thaumatin) and precipitant $\left(2 \mathrm{M} \mathrm{KNaC}_{4} \mathrm{H}_{4} \mathrm{O}_{6}\right)$ solutions in a PDMS microfluidic channel. The solutions were separated by a thin stream of buffer to avoid contact ${ }^{6}$ before forming plugs (Figure 1a). Mixing was changed by varying the total flow rate (higher flow rate corresponding to more rapid mixing in a winding channel). ${ }^{8}$ The flow rate ratios between protein, buffer, and salt solutions were kept constant. Mixed plugs were collected in a glass capillary, ${ }^{7}$ which was sealed and incubated at $18{ }^{\circ} \mathrm{C}$. The number of crystals in each plug was monitored over time.

Nucleation was sensitive to flow rate and mixing. Rapid nucleation took place at low flow velocities: i) precipitate was visible as the plugs were being mixed, and ii) these plugs yielded precipitation or showers of microcrystals after incubation for $8 \mathrm{~h}$, indicating many nucleation events per plug. At high flow velocities, no precipitation was visible, and only a few large crystals grew after $8 \mathrm{~h}$ (Figure 1) of incubation, representing only a few nucleation events in each plug.

We rationalize qualitatively the effect of mixing by considering the interface regions (interfaces) between the protein and salt solutions. Mixing by chaotic advection yields interfaces where highly concentrated protein and precipitant come in contact and interdiffuse (Figure 2). Because the precipitant diffuses more rapidly than the protein, at the interface there will be a region within which the protein concentration is still high, while it has a high

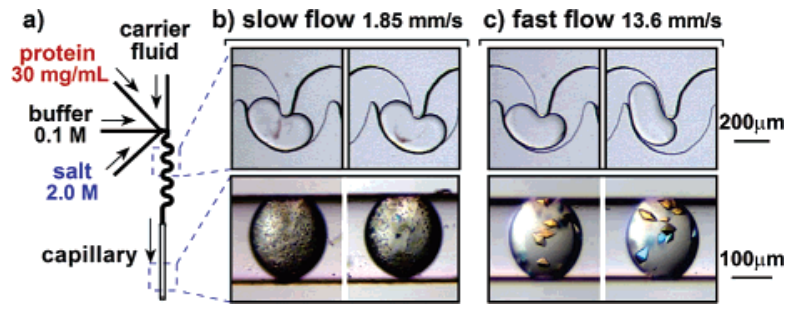

Figure 1. (a) A schematic of the microfluidic channel. (b) At low flow velocity precipitation occurred and microcrystals grew. (c) At high flow velocity no precipitation occurred and large crystals grew.

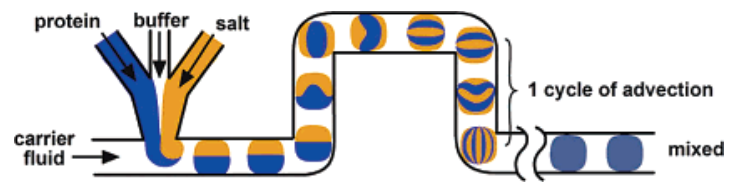

Figure 2. A simplified schematic of the stretching and folding of interfaces as plugs are being mixed by chaotic advection in a winding channel. ${ }^{8}$ See Supporting Information for experimental flow patterns.

concentration of precipitant. The nucleation rate $J$ [nuclei $\mathrm{m}^{-3} \mathrm{~s}^{-1}$ ] strongly depends ${ }^{11}$ on supersaturation $S, J \propto \exp \left[-C /(\ln S)^{2}\right] .{ }^{12}$

The supersaturation, and therefore nucleation rate at the interfaces, is much higher than it would be after mixing. Therefore, the total number of nucleation events depends on both the area and the lifetime of these interfaces. These arguments are well-known and are used as the basis for free-interface diffusion methods of protein crystallization. ${ }^{1,13}$

Varying the flow velocity varies the area as well as the lifetime of these interfaces. The interfaces stretch and fold as plugs travel through the winding channel. ${ }^{8}$ Stretching and folding increases the total area of interfaces and accelerates mixing by decreasing the diffusion distance (Figure 2). Increasing the flow velocity allows the interfaces to evolve faster and increases the total area of the interfaces that develops before mixing is complete. The lifetime of the interfaces, however, decreases at high flow velocity due to acceleration of mixing by chaotic advection.

The mixing effect can be qualitatively estimated from the product of the lifetime and total area of the interfaces (for simplicity we assume that the thickness of the interfacial region in which nucleation takes place is constant). We have made three additional approximations. First, only nucleation happening at the freshly formed interfaces is considered, rather than nucleation taking place after mixing is complete. This assumption is experimentally reasonable when the time of incubation before collecting crystal growth data is short (to reduce the importance of background nucleation from the mixed solutions). Second, every newly generated interface is fresh for only one cycle of advection, which is arbitrary, but it will cancel out as we are comparing the ratio of nucleation under different flow velocities. Removing this assumption gives qualitatively similar results. Third, the Peclet number $(P e)$ is high - convective transport dominates diffusive transport (The calculated values: Reynolds number, $\operatorname{Re}<6 ; P e>170$ ). Applying a scaling argument (with all of the necessary assumptions) 

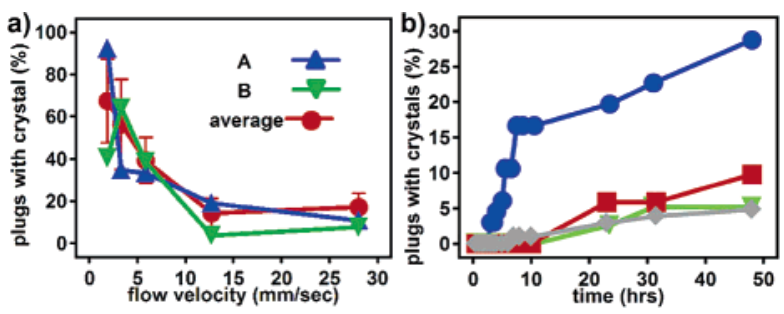

Figure 3. (a) The percentage (\%) of plugs with crystals depends on the flow velocity. The error bar for each data point was calculated from $\sim 300$ plugs from 5 to 6 different experiments. Representative data from two experiments were shown as curves A and B. (b) Effects of flow velocity and channel geometry on nucleation. (-) Slow, winding channel; (ם) slow, straight channel; ( $\boldsymbol{\nabla})$ fast, winding channel; $(\bullet)$ fast, straight channel.

developed for evaluating mixing by chaotic advection in plugs, ${ }^{8}$ the estimated number of nucleation events, $N$, is $N \propto w^{7 / 2}(\mathrm{DU})^{-1 / 2}$, where $w[\mathrm{~m}]$ is the width of the microfluidic channel, $D\left[\mathrm{~m}^{2} / \mathrm{s}\right]$ is the diffusion coefficient, and $U[\mathrm{~m} / \mathrm{s}]$ is the flow velocity (Supporting Information).

We have chosen to change the flow velocity $U$ to investigate the mixing effects, although the equation predicts a stronger dependence of nucleation on $w$. Changing $w$ would vary both the volume and the mixing rate of the plugs. Background nucleation taking place after mixing may scale with the volume of the plugs as $N \propto w^{3}$, which is difficult to differentiate from $N \propto w^{7 / 2}$. Therefore, by changing $w$ it is difficult to decouple mixing effects from background nucleation. To avoid this problem, the sizes of plugs were kept constant (as described previously ${ }^{14}$ ) within each series of experiments.

The experimental result was in qualitative agreement with the estimate. We performed experiments under lower supersaturation than in Figure 1, so only a fraction of the plugs yielded crystals after incubation of $3 \mathrm{~h}$ at $18{ }^{\circ} \mathrm{C}$ (Figure 3a). The expected weak inverse dependence of $N$ (measured as the \% of plugs with crystals) on the flow rate was observed, but the range of tested flow rates was too small to eliminate conclusively other possible estimates. Each data point was obtained by averaging results of $\sim 50$ plugs. To obtain the average curve shown in red circles, $5-6$ data points were averaged. There is scatter in these experiments because they were performed in different devices, on different days, with different solutions of the protein. Nevertheless, the data from crystallization in $\sim 1500$ plugs set up for Figure $3 \mathrm{a}$ are self-consistent.

We performed additional experiments in straight, rather than winding, channels. We wished to test if the observed difference in nucleation was a result of change in mixing, rather than other effects associated with changing flow velocity. Nucleation could have happened at the junction of inlets of protein and salt streams (Figure 1a), or could have been affected by higher shear forces at high flow rates. These effects depend on the flow velocity, rather than mixing, and should be the same in both winding and straight channels. Plugs traveling through straight channels are not mixed chaotically, there is no folding, and the area of the interface is smaller. One would expect less nucleation in straight channels, as was observed experimentally (Figure $3 b$ ). We also varied the flow velocity in straight channels (mixing does not significantly depend on flow velocity in straight channels except during formation of the plugs ${ }^{14}$ ). The observed nucleation was similar at high and low flow rates and was similar to the nucleation obtained at high flow rates in winding channels (Figure 3b). Nucleation in winding channels at low flow rates was significantly higher; the curve was characterized by the initial rapid increase in the number of crystals observed $(0-8 \mathrm{~h})$ and then slow further increase $(8-50 \mathrm{~h})$. We attribute the initial rapid increase to nucleation that occurred during mixing, followed by growth of crystals to a size that we could detect with a stereoscope $(\sim 20 \mu \mathrm{m})$. The subsequent slow increase in the number of crystals has a slope similar to that in the other three experiments in Figure 3b, suggesting that it is due to background nucleation taking place after mixing and during incubation.

The nucleation rate is affected by the area and the lifetime of the interface between the solutions, rather than simply by the mixing time (slow chaotic mixing induces nucleation more effectively than slow mixing in straight channels). This result may be used to control nucleation, using rapid chaotic mixing to eliminate formation of precipitates at high supersaturation and using slow chaotic mixing to induce nucleation at lower supersaturation. While we used a model protein (thaumatin) for the studies, we also observed these mixing effects in the crystallization of a new ubiquitination protein and obtained fewer and larger crystals at higher flow rates. This result is also consistent with the effect of mixing on autocatalytic reactions. ${ }^{15}$ The mixing effect is more pronounced at high supersaturations $\left(15-40 \mathrm{mg} / \mathrm{mL}\right.$ protein concentration); therefore, batch ${ }^{3}$ protein crystallization, which starts from high supersaturation, will be more sensitive to mixing than crystallization by vapor diffusion (no mixing effect was observed for protein concentrations below $10 \mathrm{mg} / \mathrm{mL}$ ). To qualitatively understand our experimental results at an intuitive level, we presented scaling arguments that rely on a number of assumptions. More detailed analytical or numerical theory, taking into account the effect of the buffer stream and the actual 3D mixing patterns, would be required to obtain a more quantitative description.

Acknowledgment. This work was supported by the NIH (R01EB001903), ONR Young Investigator Award (N00014-0310482), the Beckman Young Investigator program, and the MRSEC microfluidic facility funded by NSF. We thank Dr. Brenda Schulman for providing the ubiquitination protein and L. Spencer Roach for preliminary experiments.

Supporting Information Available: Experimental details and additional data (PDF). This material is available free of charge via the Internet at http://pubs.acs.org.

\section{References}

(1) McPherson, A. Crystallization of biological macromolecules; Cold Spring Harbor Laboratory Press: Cold Spring Harbor, NY, 1999.

(2) Galkin, O.; Vekilov, P. G. Proc. Natl. Acad. Sci. U.S.A. 2000, 97, 62776281. Garetz, B. A.; Aber, J. E.; Goddard, N. L.; Young, R. G.; Myerson, A. S. Phys. Rev. Lett. 1996, 77, 3475-3476. Garetz, B. A.; Matic, J.; Myerson, A. S. Phys. Rev. Lett. 2002, 89, 175501. Yau, S. T.; Vekilov, P. G. Nature 2000, 406, 494-497. Lee, A. Y.; Ulman, A.; Myerson, A. S. Langmuir 2002, 18, 5886-5898. Knezic, D.; Zaccaro, J.; Myerson, A. S. J. Phys. Chem. B 2004, 108, 10672-10677. Vekilov, P. G. Cryst. Growth Des. 2004, 4, 671-685.

(3) Chernov, A. A. J. Struct. Biol. 2003, 142, 3-21.

(4) Chen, P. C.; Chen, C. C.; Fun, M. H.; Liao, O. Y.; Jiang, J. J.; Wang, Y. S.; Chen, C. S. Chem. Eng. Technol. 2004, 27, 519-528.

(5) Suga, S.; Nagaki, A.; Yoshida, J. Chem. Commun. 2003, 354-355. Nagaki, A.; Kawamura, K.; Suga, S.; Ando, T.; Sawamoto, M.; Yoshida, J. J. Am. Chem. Soc. 2004, 126, 14702-14703.

(6) Song, H.; Tice, J. D.; Ismagilov, R. F. Angew. Chem., Int. Ed. 2003, 42, $768-772$.

(7) Zheng, B.; Roach, L. S.; Ismagilov, R. F. J. Am. Chem. Soc. 2003, 125, 11170-11171. Zheng, B.; Tice, J. D.; Roach, L. S.; Ismagilov, R. F. Angew. Chem., Int. Edit. 2004, 43, 2508-2511.

(8) Song, H.; Bringer, M. R.; Tice, J. D.; Gerdts, C. J.; Ismagilov, R. F. Appl. Phys. Lett. 2003, 83, 4664-4666. Bringer, M. R.; Gerdts, C. J.; Song, H.; Tice, J. D.; Ismagilov, R. F. Philos. Trans. R. Soc. London, Ser. A 2004, 362, 1087-1104.

(9) Stroock, A. D.; Dertinger, S. K. W.; Ajdari, A.; Mezic, I.; Stone, H. A.; Whitesides, G. M. Science 2002, 295, 647-651. Liu, R. H.; Stremler, M. A.; Sharp, K. V.; Olsen, M. G.; Santiago, J. G.; Adrian, R. J.; Aref, H. Beebe, D. J. J. Microelectromech. Syst. 2000, 9, 190-197. Ottino, J. M Wiggins, S. Science 2004, 305, 485-486.

(10) Shestopalov, I.; Tice, J. D.; Ismagilov, R. F. Lab Chip 2004, 4, 316321.

(11) Garcia-Ruiz, J. M. J. Struct. Biol. 2003, 142, 22-31.

(12) See Supporting Information for definitions and for experimental measurements of solubilities and supersaturation.

(13) Hansen, C. L.; Skordalakes, E.; Berger, J. M.; Quake, S. R. Proc. Natl. Acad. Sci. U.S.A. 2002, 99, 16531-16536. Ng, J. D.; Gavira, J. A.; GarciaRuiz, J. M. J. Struct. Biol. 2003, 142, 218-231.

(14) Tice, J. D.; Song, H.; Lyon, A. D.; Ismagilov, R. F. Langmuir 2003, 19 9127-9133.

(15) Gerdts, C. J.; Sharoyan, D. E.; Ismagilov, R. F. J. Am. Chem. Soc. 2004 $126,6327-6331$. JA052279V 